\title{
Acoustic Wave Propagation in Nanocrystalline RuCo Alloys
}

\author{
Pramod Kumar Yadawa ${ }^{1 *}$, Devraj Singh ${ }^{1}$, Dharmendra Kumar Pandey ${ }^{2}$, \\ Giridhar Mishra ${ }^{3}$, RajaRam Yadav ${ }^{3}$ \\ ${ }^{1}$ Department of Applied Physics, AMITY School of Engineering and Technology, New Delhi, India \\ ${ }^{2}$ Department of Physics, P. P. N. College, Kanpur, India \\ ${ }^{3}$ Department of Physics, University of Allahabad, Allahabad, India \\ E-mail: pkyadawa@aset.amity.edu
}

Received June 27, 2011; revised July 27, 2011; accepted August 9, 2011

\begin{abstract}
The ultrasonic properties like elastic constant, ultrasonic velocity in the hexagonal structured nanocrystalline RuCo alloys have been studied along unique axis at room temperature. The second and third order elastic constants (SOEC \& TOEC) have been calculated for these alloys using Lennard-Jones potential. The orientation dependent ultrasonic velocity has been also evaluated to study the anisotropic behaviour of these alloys. The velocities $\mathrm{V}_{\mathrm{L}}$ and $\mathrm{V}_{\mathrm{S} 1}$ have minima and maxima, respectively at $45^{\circ}$ with unique axis of the crystal, while $\mathrm{V}_{\mathrm{S2}}$ increases with the angle from unique axis. The inconsistent behaviour of angle-dependent velocities is associated to the action of second order elastic constants. Debye average ultrasonic velocities of these alloys are increasing with the angle and has maximum at $55^{\circ}$ with unique axis at room temperature. Hence, when a ultrasonic wave travels at $55^{\circ}$ with unique axis of these alloys, then the average ultrasonic velocity is found to be maximum. Elastic constants and density are mainly the affecting factor for anomalous behaviour of ultrasonic velocity in these alloys. The mechanical and ultrasonic properties of $\mathrm{Co}_{0.75} \mathrm{Ru}_{0.25}$ alloy will be better than the other compounds due to their high SOEC, ultrasonic velocity and low ultrasonic attenuation. $\mathrm{Co}_{0.75} \mathrm{Ru}_{0.25}$ alloy is more suitable for industrial and other uses, as it has the highest elastic constants and lowest ultrasonic attenuation in comparison to other of these alloys. The results of this investigation are discussed in correlation with other known thermophysical properties.
\end{abstract}

Keywords: Alloys, Elastic Properties, Ultrasonic Properties

\section{Introduction}

The structure of ruthenium $(\mathrm{Ru})$ is hexagonal close packed (hcp) whereas cobalt (Co) has three phases those are ferromagnetic; body centered cubic (bcc), face centered cubic (fcc) and hcp. The structure of Co strongly depends on the grain size. For small grain size, fcc is stable and for large grain size, the stable structure is hcp and for a distribution of grain sizes, a mixture of fcc and hcp phases exists. Huang et al. reported that the mixture of fcc and hcp phases transforms to either fcc or hcp single phase by ball milling process which introduces structural defects [1]. Alloys of $3 d$ transition metals such as $\mathrm{Ni}$, Co, and $\mathrm{Fe}$ exhibit fascinating magnetic properties. In particular, their alloys with the $4 d$ transition metals $\mathrm{Ru}$, $\mathrm{Rh}$, and Pd are the subjects of experimental and theoretical investigations. Theoretical investigations suggest an increasing ferromagnetic order in $\mathrm{Pd}, \mathrm{Rh}$, and $\mathrm{Ru}$ similar to their analog $3 d$-metals $\mathrm{Ni}$, Co, and $\mathrm{Fe}$ [2-4]. In addition, it has been discovered that there is antiferromagnetic interlayer exchange coupling and enhanced magnetoresistance in the metallic superlattices of $\mathrm{Co} / \mathrm{Ru}[5,6]$. Because the thin layer of Ru strongly antiferromagnetically couples magnetic moments of the two Co layers in an antiparallel configuration, the superlattice of $\mathrm{Ru} / \mathrm{Co}$ are finding applications in magnetic random access memory devices [7].

It has been shown that nanocrystalline particles of $\mathrm{Fe}$, $\mathrm{Co}, \mathrm{PdFe}$, and RuFe alloys were prepared using organometallic precursors and followed by pyrolysis $[5,6,8,9]$. Using the same chemical synthesis procedure and starting with organic precursor mixtures of $\mathrm{Ru}$ and $\mathrm{Co}$, RuCo alloys were synthesized over the entire compositional range. Qadri et al. studied the structural and magnetic pr- 
operties of RuCo alloys and show the existence of either the hexagonal phase or the fcc phase depending on the composition and the particle size. Also Qadri et al. reported the structural and magnetic properties of PdFe and RuFe alloys synthesized through organometallic route [10].

Ultrasonic non-destructive testing (NDT) is a useful technique that can be applied to a rang of materials for the characterization of their microstructures, the appraisal of defects and the determination of physical properties such as density, thermal conductivity and electrical resistivity. Ultrasonic measurements taken during the fabrication and heat treatment of materials can be used to ensure that the preferred microstructure is obtained and to prevent the formation of defects, including defects in welds between two different alloys. Information about the microstructure can also be used in material description studies, such as non-destructive determination of grin size Wave propagation velocity is key parameter in ultrasonic characterization and can provide information about crystallographic texture. The ultrasonic velocity is directly related to the elastic constants by the relationship $\mathrm{V}=\sqrt{(\mathrm{C} / \rho)}$, where $\mathrm{C}$ is the relevant elastic constants and $\rho$ is the density of that particular material. The elastic constants of a solid provide valuable insight into nature of atomic bonding forces and also related hardness [11,12].

There are three types of acoustic mode of lattice vibrations: one longitudinal acoustical and two transverse acoustical for hexagonal structured materials. Hence, there are three types of ultrasonic wave velocities for each direction of propagation of wave, which are well related to second order elastic constants. But all the three types of ultrasonic velocities and elastic constants of these alloys are not reported in the literature. Therefore in this paper, we have calculated the three types of ultrasonic sound velocities for the alloys $\mathrm{Co}_{0.00} \mathrm{Ru}_{1.00}$ : alloy-1; $\mathrm{Co}_{0.25} \mathrm{Ru}_{0.75}$ : alloy-2; $\mathrm{Co}_{0.40} \mathrm{Ru}_{0.60}$ : alloy-3; $\mathrm{Co}_{0.50} \mathrm{Ru}_{0.50}$ : alloy-4; $\mathrm{Co}_{0.60} \mathrm{Ru}_{0.40}$ : alloy-5; $\mathrm{Co}_{0.75} \mathrm{Ru}_{0.25}$ : alloy-6; for each direction of propagation of wave using second order elastic constants that are important for surface and structural study of these alloys. The six second order elastic constants and ten third order elastic constants are calculated using Lenard-Jones Potential that is a many body interaction potential. The results obtained are interesting for the characterization of these alloys.

\section{Theory}

\subsection{Higher Order Elastic Constants}

The second $\left(\mathrm{C}_{\mathrm{IJ}}\right)$ and third $\left(\mathrm{C}_{\mathrm{IJK}}\right)$ order elastic constants of material are defined by following expressions.

$$
\begin{aligned}
& \mathrm{C}_{\mathrm{IJ}}=\frac{\partial^{2} \mathrm{U}}{\partial \mathrm{e}_{\mathrm{I}} \partial \mathrm{e}_{\mathrm{J}}} ; \quad \text { I or } \mathrm{J}=1, \cdots, 6 \\
& \mathrm{C}_{\mathrm{IJK}}=\frac{\partial^{3} \mathrm{U}}{\partial \mathrm{e}_{\mathrm{I}} \partial \mathrm{e}_{\mathrm{J}} \partial \mathrm{e}_{\mathrm{K}}} ; \text { I or } \mathrm{J} \text { or } \mathrm{K}=1, \cdots, 6
\end{aligned}
$$

where, $U$ is elastic energy density , $e_{I}=e_{i j}$ (i or j $=x, y, z$; $\mathrm{I}=1, \cdots, 6)$ is component of strain tensor. Eqs. (1)-(2) leads six second and ten third order elastic constants (SOEC and TOEC) for the hexagonal closed packed structured materials [13].

$$
\left.\begin{array}{ll}
\mathrm{C}_{11}=24.1 p^{4} \mathrm{C}^{\prime} & \mathrm{C}_{12}=5.918 p^{4} \mathrm{C}^{\prime} \\
\mathrm{C}_{13}=1.925 p^{6} \mathrm{C}^{\prime} & \mathrm{C}_{33}=3.464 p^{8} \mathrm{C}^{\prime} \\
\mathrm{C}_{44}=2.309 p^{4} \mathrm{C}^{\prime} & \mathrm{C}_{66}=9.851 p^{4} \mathrm{C}^{\prime}
\end{array}\right\}
$$

where

$$
\begin{aligned}
& \mathrm{C}^{\prime}=\chi a / p^{5} ; \mathrm{B}=\psi a^{3} / p^{3} ; \\
& \chi=(1 / 8)\left[\left\{n b_{0}(n-m)\right\} /\left\{a^{n+4}\right\}\right] ; \\
& \psi=-\chi /\left\{6 a^{2}(m+n+6)\right\} ;
\end{aligned}
$$

$m, n=$ integer quantity; $b_{0}=$ Lennard Jones parameter. $p=c / a$ : axial ratio; " $c$ " is the height of the unit cell and " $a$ " be the basal plane distance.

\subsection{Ultrasonic Velocity}

The anisotropic behaviour of the material can be understood with the knowledge of ultrasonic velocity because the velocity is related to the second order elastic constants [13]. On the basis of mode of atomic vibration, there are three types of velocities (longitudinal, quasi shear and shear) in acoustical region [14]. These velocities vary with the direction of propagation of wave from the unique axis of hexagonal structured crystal [15]. The ultrasonic velocities as a function of angle between direction of propagation and unique axis for hexagonal structured materials are [16]:

$$
\begin{aligned}
& \mathrm{C}_{111}=126.9 p^{2} \mathrm{~B}+8.853 p^{4} \mathrm{C}^{\prime} \\
& \mathrm{C}_{113}=1.924 p^{4} \mathrm{~B}+1.155 p^{6} \mathrm{C}^{\prime} \\
& \mathrm{C}_{133}=3.695 p^{6} \mathrm{~B} \\
& \mathrm{C}_{112}=19.168 p^{2} \mathrm{~B}-1.61 p^{4} \mathrm{C}^{\prime} \\
& \mathrm{C}_{144}=2.309 p^{4} \mathrm{~B} \\
& \mathrm{C}_{123}=1.617 p^{4} \mathrm{~B}-1.155 p^{6} \mathrm{C}^{\prime} \\
& \mathrm{C}_{155}=1.539 p^{4} \mathrm{~B} \\
& \mathrm{C}_{344}=3.464 p^{6} \mathrm{~B} \\
& \mathrm{C}_{222}=101.039 p^{2} \mathrm{~B}+9.007 p^{4} \mathrm{C}^{\prime} \quad \mathrm{C}_{333}=5.196 p^{8} \mathrm{~B}
\end{aligned}
$$




$$
\begin{aligned}
\mathrm{V}_{\mathrm{L}}^{2}= & \left\{\mathrm{C}_{33} \cos ^{2} \theta+\mathrm{C}_{11} \sin ^{2} \theta+\mathrm{C}_{44}+\left\{\left[\mathrm{C}_{11} \sin ^{2} \theta-\mathrm{C}_{33} \cos ^{2} \theta+\mathrm{C}_{44}\left(\cos ^{2} \theta-\sin ^{2} \theta\right)\right]^{2}\right.\right. \\
& \left.\left.+4 \cos ^{2} \theta \sin ^{2} \theta\left(\mathrm{C}_{13}+\mathrm{C}_{44}\right)^{2}\right\}^{1 / 2}\right\} / 2 \rho \\
\mathrm{V}_{\mathrm{S} 1}^{2}= & \left\{\mathrm{C}_{33} \cos ^{2} \theta+\mathrm{C}_{11} \sin ^{2} \theta+\mathrm{C}_{44}-\left\{\left[\mathrm{C}_{11} \sin ^{2} \theta-\mathrm{C}_{33} \cos ^{2} \theta+\mathrm{C}_{44}\left(\cos ^{2} \theta-\sin ^{2} \theta\right)\right]^{2}\right.\right. \\
& \left.\left.+4 \cos ^{2} \theta \sin ^{2} \theta\left(\mathrm{C}_{13}+\mathrm{C}_{44}\right)^{2}\right\}^{1 / 2}\right\} / 2 \rho
\end{aligned}
$$

$$
\mathrm{V}_{\mathrm{S} 2}^{2}=\left\{\mathrm{C}_{44} \cos ^{2} \theta+\mathrm{C}_{66} \sin ^{2} \theta\right\} / \rho
$$

where $\mathrm{V}_{\mathrm{L}}, \mathrm{V}_{\mathrm{S} 1}$ and $\mathrm{V}_{\mathrm{S} 2}$ are longitudinal, quasi shear and pure shear wave ultrasonic velocities. Variables $\rho$ and $\theta$ represent the density of the material and angle with the unique axis of the crystal respectively. The Debye temperature $\left(T_{D}\right)$ is an important physical parameter for the characterization of materials, which is well related to the Debye average velocity $\left(\mathrm{V}_{\mathrm{D}}\right)$.

$$
\begin{aligned}
\mathrm{T}_{\mathrm{D}} & =\frac{\hbar \mathrm{V}_{\mathrm{D}}\left(6 \pi^{2} n_{a}\right)^{1 / 3}}{k_{B}} \\
\text { here } \mathrm{V}_{\mathrm{D}} & =\left\{\frac{1}{3}\left(\frac{1}{\mathrm{~V}_{\mathrm{L}}^{3}}+\frac{1}{\mathrm{~V}_{\mathrm{S} 1}^{3}}+\frac{1}{\mathrm{~V}_{\mathrm{S} 2}^{3}}\right)\right\}^{-1 / 3}
\end{aligned}
$$

where $\hbar$ is quantum of action and is equal to Planck's constant divided by $2 \pi ; k_{B}$ is Boltzmann Constant; $n_{a}$ is atom concentration.

The above formulae have been used for the evaluation of ultrasonic velocity and related parameters for the selected materials.

\section{Results}

The unit cell parameters " $a$ " for these six alloys (1, 2, 3,
4, 5 and 6) are $2.705 \AA, 2.682 \AA, 2.655 \AA, 2.606 \AA$, $2.595 \AA$ and $2.565 \AA$ respectively and axial ratio " $p$ " for these alloys are 1.583, 1.589, 1.590, 1.613, 1.616 and 1.623 respectively [10]. The value of $m, n$ and $b_{0}$ for these alloys are 6, 7 and $9.9 \times 10^{-64} \mathrm{erg} \mathrm{cm}^{7}$ correspondingly. The second and third order elastic constants (SOE $\mathrm{C}$ and TOEC) have been calculated for RuCo alloys using Eqs. (3a) and (3b) and are presented in Table 1. The calculated oriented dependent ultrasonic velocities at 300 $\mathrm{K}$ are shown in Figures 1-4.

\section{Discussions}

The elastic constants are important since they are related to hardness and are used for the determination of the ultrasonic velocity. It is obvious from Table 1 that, there is good agreement between the present values of SOEC and TOEC with other of alloy: 1 (i.e. Ru). Hence present values of elastic constants are justified. The bulk modulus (B) for these alloys can be calculated with the formula $\mathrm{B}=2\left(\mathrm{C}_{11}+\mathrm{C}_{12}+2 \mathrm{C}_{13}+\mathrm{C}_{33} / 2\right) / 9$. The evaluated $\mathrm{B}$

\begin{tabular}{|c|c|c|c|c|c|c|c|c|c|c|}
\hline Alloys & $\mathrm{C}_{11}$ & $\mathrm{C}_{12}$ & $\mathrm{C}_{13}$ & $\mathrm{C}_{33}$ & $\mathrm{C}_{44}$ & $\mathrm{C}_{66}$ & B & & & \\
\hline 1 & 6.28 & 1.54 & 1.26 & 5.67 & 1.51 & 2.46 & 2.91 & & & \\
\hline 2 & 6.83 & 1.68 & 1.38 & 6.26 & 1.65 & 2.68 & 3.19 & & & \\
\hline 3 & 7.53 & 1.85 & 1.52 & 6.92 & 1.82 & 2.95 & 3.52 & & & \\
\hline 4 & 8.95 & 2.20 & 1.86 & 8.71 & 2.23 & 3.51 & 4.27 & & & \\
\hline 5 & 9.34 & 2.29 & 1.95 & 9.15 & 2.34 & 3.66 & 4.46 & & & \\
\hline 6 & 10.43 & 2.56 & 2.19 & 10.40 & 2.63 & 4.09 & 5.02 & & & \\
\hline $\mathrm{Ru}[17]$ & 6.28 & 1.54 & 1.26 & 5.65 & 1.51 & 2.46 & 2.91 & & & \\
\hline Alloys & $\mathrm{C}_{111}$ & $\mathrm{C}_{112}$ & $\mathrm{C}_{113}$ & $\mathrm{C}_{123}$ & $\mathrm{C}_{133}$ & $\mathrm{C}_{344}$ & $\mathrm{C}_{144}$ & $\mathrm{C}_{155}$ & $\mathrm{C}_{222}$ & $\mathrm{C}_{333}$ \\
\hline 1 & -102.44 & -16.24 & -3.22 & -4.10 & -19.14 & -17.95 & -4.77 & -3.18 & -81.05 & -67.44 \\
\hline 2 & -111.37 & -17.66 & -3.53 & -4.49 & -21.15 & -19.82 & -5.23 & -3.48 & -88.12 & -75.09 \\
\hline 3 & -122.77 & -19.46 & -3.90 & -4.96 & -23.38 & -21.92 & -5.78 & -3.85 & -97.14 & -83.15 \\
\hline 4 & -145.93 & -23.14 & -4.77 & -6.06 & -29.41 & -27.57 & -7.06 & -4.71 & -115.47 & -107.61 \\
\hline 5 & -152.25 & -24.14 & -4.99 & -6.35 & -30.91 & -28.98 & -7.40 & -4.93 & -120.46 & -113.52 \\
\hline 6 & -170.08 & -26.97 & -5.63 & -7.15 & -35.14 & -32.95 & -8.34 & -5.56 & -134.57 & -130.20 \\
\hline $\mathrm{Ru}[17]$ & -102.40 & -16.24 & -3.22 & -4.09 & -19.10 & -17.91 & -4.77 & -3.18 & -8.03 & -67.21 \\
\hline
\end{tabular}
for these alloys is presented in Table 1 . It is obvious from Table 1, that there is good agreement between the calculated values from this study and the previously re ported values for "B" for Ru [17]. Thus our theoretical approach for the calculation of second order elastic constants

Table 1. SOEC, TOEC and Bulk modulus (B) (in the unit of $10^{11} \mathrm{~N} \cdot \mathrm{m}^{-2)}$ of RuCo alloys at room temperature. 


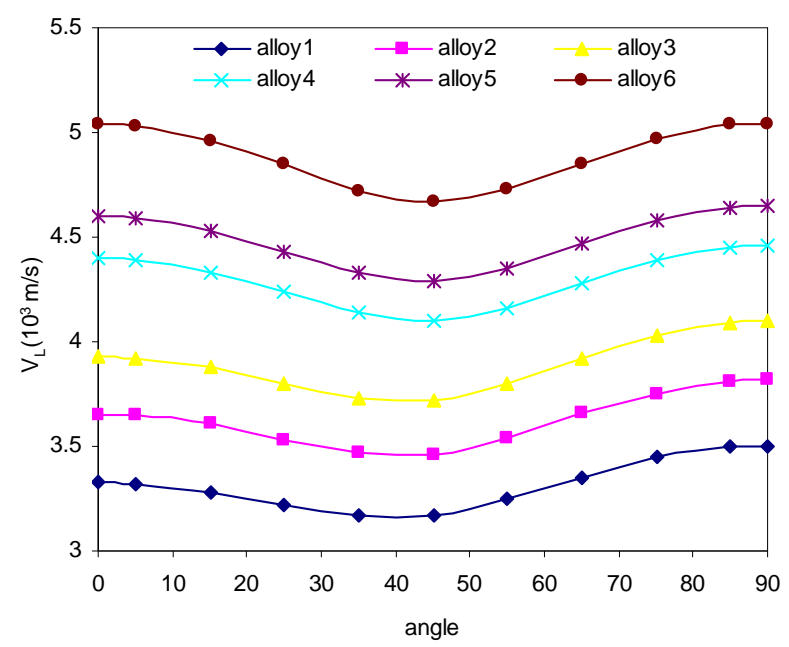

Figure 1. $V_{L}$ vs angle with unique axis of crystal.

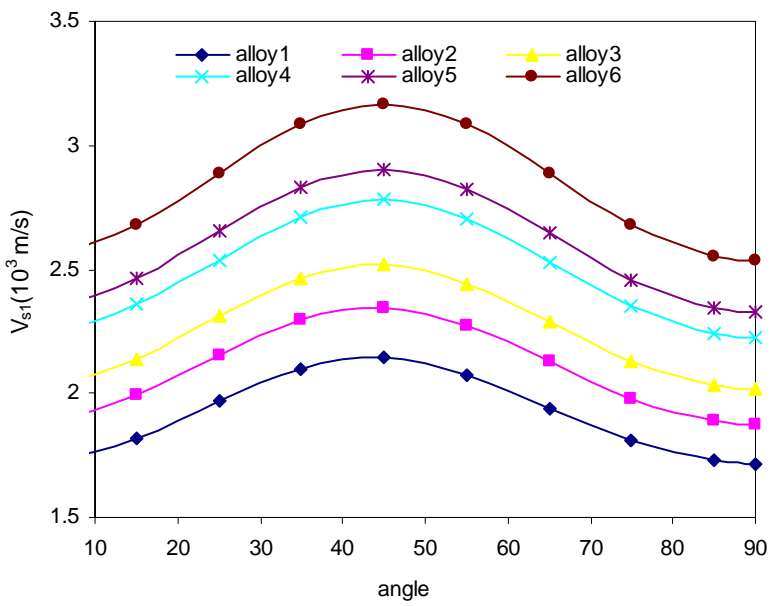

Figure 2. $\mathrm{V}_{\mathrm{S} 1}$ vs angle with unique axis of crystal.

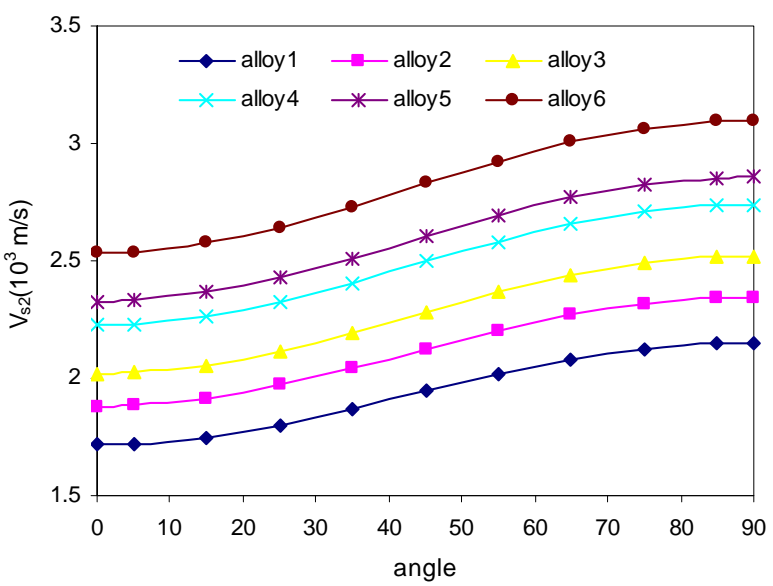

Figure 3. $\mathrm{V}_{\mathrm{S} 2}$ vs angle with unique axis of crystal.

for hexagonal structured alloys at room temperature is well justified. Hence applied theory for the evaluation of higher order elastic constants at room temperature is justified. All the SOEC and TOEC for these alloys are found to be higher than those of Mo-Ru-Rh-Pd alloys [13]. It can be also seen from Table 1, that the SOEC and TOEC are found to be increasing from alloys: 1-6 due to large grain size of hcp Co metals.

Figures 1-3 show that the velocities $V_{L}$ and $V_{S 1}$ have minima and maxima respectively at $45^{\circ}$ with the unique axis of the crystal while $V_{\mathrm{S} 2}$ increases with the angle from the unique axis. Anomalous behaviour of angle dependent velocities is correlated to the behaviour of second order elastic constants. The nature of the angle dependent velocity curves in the present work is the same as the nature of angle dependent velocity curve for others hexagonal wurtzite structured materials [13,1518]. Thus our angle dependency of the velocities for these nanocrystalline wurtzite structured alloys is justified.

Figures 1-3 indicate that the magnitude of acoustic velocity is larger for alloy: 6 and smaller for alloy: 1 . The respective smaller magnitude of acoustical velocity in alloy: 1 is due to its higher gravitational density. The larger longitudinal acoustical velocity along the [001] direction ( $\theta=0^{\circ}$ with unique axis) for alloy: 6 are due to the highest value of $\mathrm{C}_{33}$ second order elastic constants. The shear wave is also called the surface wave. Therefore the acoustical velocities $V_{\mathrm{S} 1}$ and $V_{\mathrm{S} 2}$ are the surface wave velocity. $V_{S 1}$ and $V_{S 2}$ have the same value for acoustic wave propagation along $\theta=0^{\circ}$ while variation is obtained between them for other directions of propagation (Figures 2 and 3). This implies that the [001] direction is the direction of symmetry for these alloys. Debye average velocities $\left(\mathrm{V}_{\mathrm{D}}\right)$ of these alloys are increasing with the angle and have maxima at $55^{\circ}$ at $300 \mathrm{~K}$ (Figure 4). Since $V_{D}$ is calculated using $V_{L}, V_{S 1}$ and $V_{S 2}$ with Equation (8), therefore the orientation variation of $V_{D}$ follows the combined effect of temperature variation of $\mathrm{V}_{\mathrm{L}}, \mathrm{V}_{\mathrm{S} 1}$ and $\mathrm{V}_{\mathrm{S} 2}$. The maximum in $\mathrm{V}_{\mathrm{D}}$ at $55^{\circ}$ is due to a

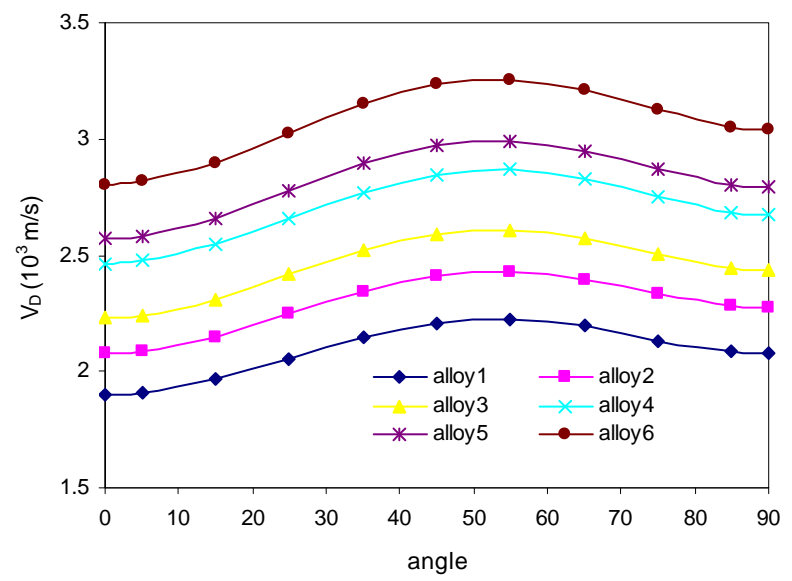

Figure 4. $V_{D}$ vs angle with unique axis of crystal. 
significant increase in longitudinal and pure shear wave velocities and a decrease in quasi-shear wave velocity. Thus it can be concluded that when a sound wave travels at $55^{\circ}$ with the unique axis of these crystals then the average sound wave velocity is maximum. The orientation dependent ultrasonic velocity $\mathrm{V}_{\mathrm{L}}, \mathrm{V}_{\mathrm{S} 1}$ and $\mathrm{V}_{\mathrm{S} 2}$ and Debye average velocity $V_{D}$ in alloy: 1 is same as pure hcp $R u$ [17]. Since the Debye average velocity is calculated using the constituent velocities $\mathrm{V}_{\mathrm{L}}, \mathrm{V}_{\mathrm{S} 1}$ and $\mathrm{V}_{\mathrm{S} 2}$, hence $\mathrm{a}$ good resemblance in $V_{D}$ implies that our calculated velocities are correct.

It can be seen that from Figures 1-4 alloy: 6 has maximum velocity and alloy: 1 has least velocity for all angles of the crystals. Since ultrasonic attenuation A $\propto \mathrm{V}^{-3}$ and velocity is the largest for alloy: 6 among others thus the attenuation A should be smallest and material should be most ductile. The minimum ultrasonic attenuation for alloy: 6 justify its quite stable hcp structure state. Also alloy: 6 has maximum elastic constants and bulk modulus among others. Hence alloy: $6\left(\mathrm{Co}_{0.75} \mathrm{Ru}_{0.25}\right)$ is more ductile, stable and contain few defects in the crystal structure in comparison to other alloys.

The pulse echo technique (PET) can be used for the measurement of ultrasonic parameters, because it avoids heat loss and scattering loss. Elastic constants and other ultrasonic parameters of binary Fe-Co and Fe-Ru alloys have been determined by a pulse echo technique [19].

\section{Conclusions}

Based on the above discussion it is worthwhile to state that:

1) Present method to evaluate second and third-order elastic constants involving many body interaction potential for hexagonal wurtzite crystal structured materials is correct.

2) Elastic constants and density are mainly the affecting factor for anomalous behaviour of acoustical velocity in these alloys.

3) When a sound wave travels at $55^{\circ}$ with the unique axis of these crystals then the average sound wave velocity is maximum. Since the Debye average velocity is calculated using the constituent velocities $V_{L}, V_{S 1}$ and $\mathrm{V}_{\mathrm{S} 2}$, hence a good resemblance in $\mathrm{V}_{\mathrm{D}}$ implies that our calculated velocities are correct.

4) The [001] direction is the direction of symmetry for these alloys as they have the same quasi-shear and pure shear wave velocities.

5) $\mathrm{Co}_{0.75} \mathrm{Ru}_{0.25}$ (alloy-6) is more suitable for industrial and other uses, as it has the highest elastic constants as well as wave velocity and lowest attenuation in comparison to other chosen alloys.

6) The mechanical and ultrasonic properties of $\mathrm{Co}_{0.75} \mathrm{Ru}_{0.25}$ alloy will be better than the other compounds due to their high SOEC and low ultrasonic attenuation.

The results obtained in this investigation can be used for further study of these alloys. Our whole theoretical approach can be applied to the evaluation of ultrasonic velocities and related parameters to study the microstructural properties of h.c.p. structured materials.

\section{References}

[1] J. Y. Huang, Y. K. Wu and H. Q. Ye, "Response to Comment on Phase Transformation of Cobalt Induced by Ball Milling," Applied Physics Letters, Vol. 67, No. 13, 1995, pp. 1944-1945. doi:10.1063/1.114577

[2] J. Crangle and W. R. Scott, "Dilute Ferromagnetic Alloys,” Journal of Applied Physics, Vol. 36, No. 3, 1965, pp. 921-928. doi:10.1063/1.1714264

[3] J. Neuwenhuys, "Magnetic Behaviour of Cobalt, Iron and Manganese Dissolved in Palladium," Advances in Physics, Vol. 24, No. 4, 1975, pp. 515-591. doi:10.1080/00018737500101461

[4] P. H. Dederich, R. Zeller, H. Akai and H. Ebert, "Ab-Initio Calculations of the Electronic Structure of Impurities and Alloys of Ferromagnetic Transition Metals," Journal of Magnetism and Magnetic Materials, Vol. 100, No. 1-3, 1991, pp. 241-260. doi:10.1016/0304-8853(91)90823-S

[5] S. S. P. Parkin, N. More and K. P. Roche, “Ab-Initio Calculations of the Electronic Structure of Impurities and Alloys of Ferromagnetic Transition Metals," Physical Review Letters, Vol. 64, 1990, pp. 2304-2307. doi:10.1103/PhysRevLett.64.2304

[6] Y. Y. Huang, G. P. Felcher and S. S. P. Parkin, "AntiFerromagnetic and Ferromagnetic Order in Co/Ru Multilayers," Journal of Magnetism and Magnetic Materials, Vol. 99, No. 1-3, 1991, pp. L31-L38. doi:10.1016/0304-8853(91)90044-B

[7] S. S. P. Parkin, K. P. Roche, M. G. Samant, P. M. Rice, R. B. Beyers, R. E. Scheueriein, E. J. O’Sullivan, S. L. Brown, J. Bucchigano, D. W. Abraham, Y. Lu, M. Rooks, P. L. Trouilloud, R. A. Warner and W. J. Gallagher, "Exchange-Biased Magnetic Tunnel Junctions and Application to Nonvolatile Magnetic Random Access Memory,” Journal of Applied Physics, Vol. 85, No. 8, 1999, pp. 5828-5833. doi:10.1063/1.369932

[8] W. Koester and E. Horn, "Characterization of Cobalt, Rhenium, Ruthenium, Osmium, Rhodium and Iridium," Journal of Materials Chemistry, Vol. 43, No. 12, 1952, pp. 444-449.

[9] T. M. Keller and S. B. Qadri, "Ferrocenylethynylbenzenes as Precursors to in Situ Synthesis of Carbon Nanotube and Fe Nanoparticle Compositions," Chemistry of Materials, Vol. 16, No. 6, 2004, pp.1091-1097. doi:10.1021/cm030624e

[10] S. B. Qadri, T. M. Keller, M. Laskoski, C. A. Little, M. S. Osofsky and H. R. Khan, "Structural and Magnetic Prop- 
erties of Nanocrystalline RuCo Alloys,” Applied Physics Letters, Vol. 91, 2007, pp. 214101: (1-3).

[11] D. Singh and P. K. Yadawa, "Effect of Platinum Addition to Coinage Metals on Their Ultrasonic Properties," Platinum Metals Review, Vol. 54, No. 3, 2010, pp. 169-176. doi:10.1595/147106710X500602

[12] P. K. Yadawa, "Ultrasonic Wave Propagation in Cadmium Chalcogenides Compounds," Multidiscipline Modeling in Materials and Structures, Vol. 7, No. 1, 2011, pp. 63-72. doi:10.1108/15736101111141449

[13] A. K. Yadav, R. R. Yadav, D. K. Pandey and D. Singh, "Ultrasonic Study of Fission Products Precipitated in the Nuclear Fuel,” Materials Letters, Vol. 62, 2008, pp. 3258-3261. doi:10.1016/j.matlet.2008.02.036

[14] C. Oligschleger, R. O. Jones, S. M. Reimann and H. R. Schober, "Model Interatomic Potential for Simulations in Selenium," Physical Review B, Vol. 53, 1996, pp. 6165-6173. doi:10.1103/PhysRevB.53.6165
[15] P. K. Yadawa, "Acoustic Wave Propagation in $\mathrm{Ni}_{3} \mathrm{R}(\mathrm{R}=$ Mo, Nb, Ta) Compounds,” Pramana, Vol. 76, No. 4, 2011, pp. 613-619. doi:10.1007/s12043-011-0066-7

[16] P. K. Yadawa, "Ultrasonic Characterization of Ceramic Material Titanium Diboride,” Ceramics-Silikaty, Vol. 55, No. 2, 2011, pp. 127-133.

[17] D. K. Pandey, D. Singh and P. K. Yadawa, "Ultrasonic Study of Osmium and Ruthenium,” Platinum Metals Review, Vol. 9, No. 2, 2009, pp. 91-97. doi:10.1595/147106709X430927

[18] D. K. Pandey, P. K. Yadawa and R. R. Yadav, "Ultrasonic Properties of Hexagonal ZnS at Nanoscale,” Materials Letters, Vol. 61, 2007, pp. 5194-5198. doi:10.1016/j.matlet.2007.04.028

[19] G. R. Speich, A. J. Schwoeble and W. C. Leslie, "Elastic Constants of Binary Iron-Base Alloys,” Metallurgical and Materials Transctions B, Vol. 3, 1972, pp. 2031-2037. 